Article

\title{
An Intuitionistic Evidential Method for Weight Determination in FMEA Based on Belief Entropy
}

\author{
Zeyi Liu and Fuyuan Xiao *(1) \\ School of Computer and Information Science, Southwest University, Chongqing 400715, China; \\ liuzeyiswu@163.com \\ * Correspondence: xiaofuyuan@swu.edu.cn
}

Received: 23 January 2019; Accepted: 20 February 2019; Published: 22 February 2019

\begin{abstract}
Failure Mode and Effects Analysis (FMEA) has been regarded as an effective analysis approach to identify and rank the potential failure modes in many applications. However, how to determine the weights of team members appropriately, with the impact factor of domain experts' uncertainty in decision-making of FMEA, is still an open issue. In this paper, a new method to determine the weights of team members, which combines evidence theory, intuitionistic fuzzy sets (IFSs) and belief entropy, is proposed to analyze the failure modes. One of the advantages of the presented model is that the uncertainty of experts in the decision-making process is taken into consideration. The proposed method is data driven with objective and reasonable properties, which considers the risk of weights more completely. A numerical example is shown to illustrate the feasibility and availability of the proposed method.
\end{abstract}

Keywords: failure mode and effects analysis; evidence theory; belief entropy; intuitionistic fuzzy set; evidence distance; weight

\section{Introduction}

Failure Mode and Effects Analysis (FMEA) has received attention from many researchers [1-7], and it can evaluate and analyze various risks in order to reduce these risks to acceptable levels or directly eliminate them. Moreover, FMEA is a very complex system so that information fusion technology is used in evaluation processes, such as evidence theory [8,9] and D number [10]. Since the uncertainty information is inevitable in FMEA, some methods have been widely used, such as Dempster-Shafer evidence theory and so on [11-13].

Though FMEA has been used in practice for many years, how to determine the weights of risk factors and team members is still an open issue. In order to define the weights more reasonably, some scholars have proposed many methods. The intuitionistic fuzzy entropy is introduced by Lei and Wang [14] to determine the weights of risk factors, while, in [15], the weights of risk factors are calculated by the objective weights. While Boran et al. [16] determined the subjective weights of risk factors. In the method proposed in [17], the weights of risk factors are simply determined by the weights calculation proposed by Boran et al. [16], although the intuitionistic fuzzy set (IFS) model is efficient to deal with FMEA [18]. However, existing methods do not take the uncertainty into consideration for the relative importance of team members.

In recent years, the relative concept of intelligence has been paid great attention due to the simulation of human intelligence $[19,20]$. As a result, it is reasonable to model experts' uncertainty in the process of decision-making in FMEA, which is important to improve the intelligent degree of the evaluation system. Thus, the measurement of uncertainty should also be regarded as content worth exploring. The related research of uncertainty metrics has been heavily discussed [21-24]. For probability distributions, Shannon entropy is efficient to handle the uncertainty [25]. However, 
it can not deal with the uncertainty of basic probability assignment (BPA) in Dempster-Shafer evidence theory [26]. To address this issue, a new belief entropy, named Deng entropy, is presented [26]. In recent years, the belief entropy has been widely used in many fields $[27,28]$.

In this paper, a hybrid weights determination of team members in the FMEA model is proposed based on the evidence distance [29] and the belief entropy [26]. The evidence distance is to measure the degree of conflict for all team members, and the belief entropy is used to model the domain experts' uncertainty in FMEA. With the combination of evidence distance, the new weights of team members are obtained, which makes the final rank of failure modes be more effective and reasonable.

The rest of this paper is organized as follows. In Section 2, some basic definitions about the evidence theory, IFS, and belief entropy are briefly introduced. In Section 3, the new method to determine the weights of team members is proposed. In Section 4, a numerical example and the computational process are illustrated. Furthermore, the comparisons and discussion have been also mentioned. In Section 5, some conclusions of the proposed method are drawn.

\section{Preliminaries}

In this section, some basic concepts which include evidence theory, intuitionistic fuzzy sets and belief entropy will be introduced.

\subsection{Evidence Theory}

Evidence theory was developed by Shafer and was firstly proposed by Dempster [30], thus it is also called D-S evidence theory. Recently, theoretical research on evidence theory has played a very important role in many applications such as decision-making [31-34], complex networks [35,36], fault diagnosis [37-41], classification [42,43] and so on [44-47].

In evidence theory [30], there is a fixed set of $N$ mutually exclusive and exhaustive elements, called the frame of discernment, which is symbolized by $\Omega=\left\{H_{1}, H_{2}, H_{3} \cdots H_{N}\right\} . P(\Omega)$ is denoted as the power set composed of $2^{N}$ elements of $\Omega$. Each element of $2^{N}$ represents a proposition [48].

Definition 1. A basic probability assignment (BPA) is a function. The range is from $P(\Omega)$ to $[0,1]$, which is defined by [30]

$$
m: P(\Omega) \rightarrow[0,1], A \mapsto m(A)
$$

and it must satisfy the following conditions:

$$
\sum_{A \in P(\Omega)} m(A)=1, m(\varnothing)=0 .
$$

The mass $m(A)$ indicates the strength of the evidence's support for $A$, while $m(\Omega)$ is represented as the uncertainty of evidence. If $m(\Omega)=1$, no useful information from the evidence exists.

The basic probability assignment (BPA) function, plausibility function (PF), belief function (BF) and other trust quantization functions are described as follows. Each function has a clear definition with physical meaning and there are some corresponding relationships.

Definition 2. Given a BPA $m$, for a proposition $A \subseteq \Omega$, the belief function Bel: $2^{\Omega} \rightarrow[0,1]$ is defined as [30]

$$
\operatorname{Bel}(A)=\sum_{B \subseteq A} m(B) .
$$

The plausibility function Pl: $2^{\Omega} \rightarrow[0,1]$ is defined as

$$
\operatorname{Pl}(A)=1-\operatorname{Bel}(\bar{A})=\sum_{B \cap A \neq \varnothing} m(B),
$$


where $\bar{A}=\Omega-A$. The quantity of $\operatorname{Bel}(A)$ can be seen as a measure of people's belief that the hypothesis $A$ is true and can be viewed as a lower limit function on the probability of $A$. The plausibility $\mathrm{Pl}(A)$ can be interpreted as the degree that we absolutely believe in $A$ and can be seen as an upper limit function on the probability of $A$.

Based on the classical evidence theory, the combination rules to aggregate the multiple sources are defined as follows.

Definition 3. Assume that there are two bodies of evidence $m_{1}$ and $m_{2}$ defined on $\Omega$, respectively, $m_{1}$ and $m_{2}$ can be combined with Dempster's orthogonal rule as follows [48,49]:

$$
m_{1} \oplus m_{2}=m(A)=\frac{\sum_{B \cap C=A} m_{1}(B) m_{2}(C)}{1-K},
$$

where

$$
K=\sum_{B \cap C=\varnothing} m_{1}(B) m_{2}(C),
$$

where $K$ (conflict coefficient) is called the degree of conflict which measures the degree of conflict between $m_{1}$ and $m_{2}$. If $K=0$, it means that there is no conflict between $m_{1}$ and $m_{2}$, and if $K=1$, it means that $m_{1}$ and $m_{2}$ is a complete contradiction. In recent years, more and more scholars have paid attention to improve the method of combination rules [50,51].

\subsection{Evidence Distance}

Evidence distance is regarded as an effective method to measure the conflict of evidence. Here are some of the basic concepts:

Definition 4. Assume that $m_{1}$ and $m_{2}$ are two BPAs defined on the same frame of discernment $\Omega$, which contains $N$ mutually exclusive and exhaustive hypotheses. Namely, it can be expressed as $\Omega=\left\{H_{1}, H_{2}, \ldots, H_{N}\right\}$. The basic definition can be defined as follows [29]:

$$
d_{B P A}\left(m_{1}, m_{2}\right)=\sqrt{\frac{1}{2}\left(\vec{m}_{1}-\vec{m}_{2}\right)^{T} \underset{=}{D}\left(\overrightarrow{m_{1}}-\overrightarrow{m_{2}}\right)},
$$

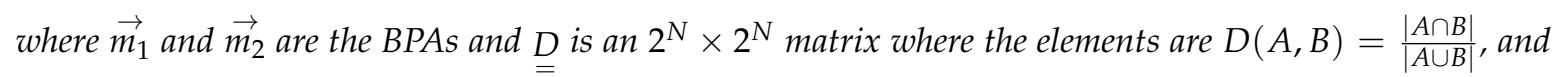
$A, B \subseteq U$. Another way to represent $\overline{\bar{d}}_{B P A}$ is

$$
d_{B P A}\left(m_{1}, m_{2}\right)=\sqrt{\frac{1}{2}\left(\left\|\overrightarrow{m_{1}}\right\|^{2}+\left\|\overrightarrow{m_{2}}\right\|^{2}-2<\overrightarrow{m_{1}}, \overrightarrow{m_{2}}>\right)},
$$

where $\left\|\overrightarrow{m_{1}}\right\|^{2}=\langle\vec{m}, \vec{m}\rangle$, and $\left.<\overrightarrow{m_{1}}, \overrightarrow{m_{2}}\right\rangle$ is the scalar product defined by

$$
<\overrightarrow{m_{1}}, \overrightarrow{m_{2}}>=\sum_{i=1}^{2^{n}} \sum_{j=1}^{2^{n}} m_{1}\left(A_{i}\right) m_{2}\left(A_{j}\right) \frac{\left|A_{i} \cap A_{j}\right|}{\left|A_{i} \cup A_{j}\right|}
$$

with $A_{i}, A_{j} \in P(\Omega), i, j=1,2, \ldots, 2^{N}$.

To combine the multiple source of evaluation and better solve the combination issues of highly conflicting evidence, the weighted average method is proposed by Deng et al. [52].

\subsection{Intuitionistic Fuzzy Set}

Since the intuitionistic fuzzy set (IFS) is proposed by Atanassov as a generalization of fuzzy sets in 1986, the aggregation of fuzzy sets and IFS theory have received a lot of attention in the past few years $[53,54]$. In a classical fuzzy sets theory, the relationship between each set is only Belong to or Not 
Belong to. The central idea of the traditional fuzzy set is to expand the characteristic function to the closed interval $[0,1]$. Based on it, the intuitionistic fuzzy sets were introduced to express the uncertain information better. Here are some of the basic definitions:

Definition 5. An intuitionistic fuzzy set (IFS) $A$ on the space $X$ is defined by two functions, $A=\left\langle A^{+}, A^{-}\right\rangle$, $A^{+}(x)$ can be represented by the degree of membership of $x$ in $A$ and $A^{-}(x)$ can be represented by the degree of nonmembership of $x$ in A. Furthermore, it satisfies the condition that [55]

$$
0 \leq A^{+}(x)+A^{-}(x) \leq 1
$$

where $A^{+}(x) \in[0,1]$ and $A^{-}(x) \in[0,1]$.

The degree of hesitancy of $x$ is defined as

$$
\operatorname{Hes}(x)=1-\left(A^{+}(x)+A^{-}(x)\right) .
$$

Thus, the membership grade of $x$ in the IFS A can be expressed by the tuple $A(x)=\left\langle A^{+}(x), A^{-}(x)>\right.$.

With the development of IFS and evidence, the relationship between these two mathematical models has been investigated more and more. Here is a brief introduction.

Assume that there exists an IFS $A=\left\{\left\langle x, \mu_{A}(x), v_{A}(x)\right\rangle \mid x \in X\right\}$. The three kinds of variables are differentiated and denoted $x \in A, x \notin A$, and the situation of hesitation when both the two hypotheses can not be approved or rejected. In this case above, the relationship between IFS and evidence theory by mathematical modelling can be found, which can be expressed that [56]

$$
\begin{gathered}
m(\text { Yes })=\mu_{A}(x), \\
m(N o)=v_{A}(x), \\
m(Y e s, N o)=\pi_{A}(x) .
\end{gathered}
$$

Recalling the evidence theory, the IFS $A$ can also be expressed as another form [56]

$$
A=\left\{<x, B I_{\mathrm{A}}(x)>\mid x \in X\right\},
$$

where

$$
\begin{aligned}
& \operatorname{Bel}_{A}(x)=m(\text { Yes })=\mu_{A}(x), \\
& P l_{A}(x)=m(\text { Yes })+m(\text { Yes, No }) \\
& =\mu_{A}(x)+\pi_{A}(x) \\
& =1-v_{A}(x) .
\end{aligned}
$$

Thus, the belief interval of proposition $A$ is defined as follows:

$$
B I_{A}(x)=\left[B e l_{A}(x), P l_{A}(x)\right]
$$

Definition 6. Assume that there exist two alternatives $x_{i}$ and $x_{j}$. Based on the conversion process, the belief interval for those two alternatives can be defined as follows [57]:

$$
P\left(x_{i}>x_{j}\right)=\frac{\max \left\{0, \operatorname{Pl}\left(x_{i}\right)-\operatorname{Bel}\left(x_{j}\right)\right\}-\max \left\{0, \operatorname{Bel}\left(x_{i}\right)-\operatorname{Pl}\left(x_{j}\right)\right\}}{\left[\operatorname{Pl}\left(x_{i}\right)-\operatorname{Bel}\left(x_{i}\right)\right]+\left[\operatorname{Pl}\left(x_{j}\right)-\operatorname{Bel}\left(x_{j}\right)\right]},
$$

where $P\left(x_{i}>x_{j}\right)$ expressed the degree of possibility of $x_{i}>x_{j}$. 


\subsection{Belief Entropy}

In the classical information science, Shannon entropy has been used in many applications [58]. Here are some of the basic definitions:

Definition 7. Shannon entropy is defined as [25]

$$
H=-\sum_{i=1}^{N} p_{i} \log _{b} p_{i}
$$

where $N$ is the number of basic states, $p_{i}$ denotes the probability of state $i$, and $p_{i}$ satisfies

$$
\sum_{i=1}^{N} p_{i}=1
$$

If the unit information is bit, then $b=2$, Shannon entropy is expressed as

$$
H=-\sum_{i=1}^{N} p_{i} \log _{2} p_{i}
$$

However, since Dempster-Shafer evidence theory has been widely used in many fields, the method to measure the uncertainty in evidence theory is still an issue worth exploring. To measure the uncertain information better, a belief entropy, named as Deng entropy, is presented to deal with uncertainty measure of BPA based on Shannon entropy [25]. Here are some of the basic definitions:

Definition 8. In frame of discernment $X$, the belief entropy is defined as [26]

$$
E_{d}(m)=-\sum_{A \subseteq X} m(A) \log _{2} \frac{m(A)}{2^{|A|}-1}
$$

where $|A|$ is the cardinality of the proposition $A$ and $E_{d}(m)$ expresses the belief entropy for basic possibility assignments. In particular, the belief entropy can definitely degenerate to the Shannon entropy if the belief is only assigned to single elements. With the development of evidence theory, the belief entropy has been more and more researched $[59,60]$.

\section{The Proposed Method}

In this section, a new method to determine the weights of team members based on the evidence theory, intuitionistic fuzzy sets and belief entropy is proposed to rank the failure modes. The function of Failure Mode and Effects Analysis (FMEA) team members is to assess the risk factors with linguistic variables, such as very low, low, medium, high, very high and so on. Assume that there are $k$ cross-functional team members $T M_{k}(k=1, \ldots, p)$ in an FMEA team; after discussing them, the experts prioritize $i$ potential failure modes $F M_{i}(i=1, \ldots, m)$. Each failure mode is evaluated on the $j$ risk factor $R F_{i}(j=1,2,3) . \lambda_{i j}^{k}$ is the weight of decision makers which reflects the relative importance of the $k$ th decision maker with respect to the $j$ th risk factors for the $i$ th potential failure modes. In addition, intuitionistic fuzzy numbers [61], which are represented by the ordered pairs of membership degrees and non-memberships corresponding to the intuitionistic fuzzy sets, is used to simply express the relevant conversion process.

Assume that the IFN $\alpha_{i j}^{k}=\left(\mu_{i j}^{k}, v_{i j}^{k}\right)$ is provided by $T M_{k}$ on the assessment of $F M_{i}$ for $R F_{j}$. The proposed method consists of eleven steps. In addition, the flowchart of the proposed approach is shown in Figure 1. 


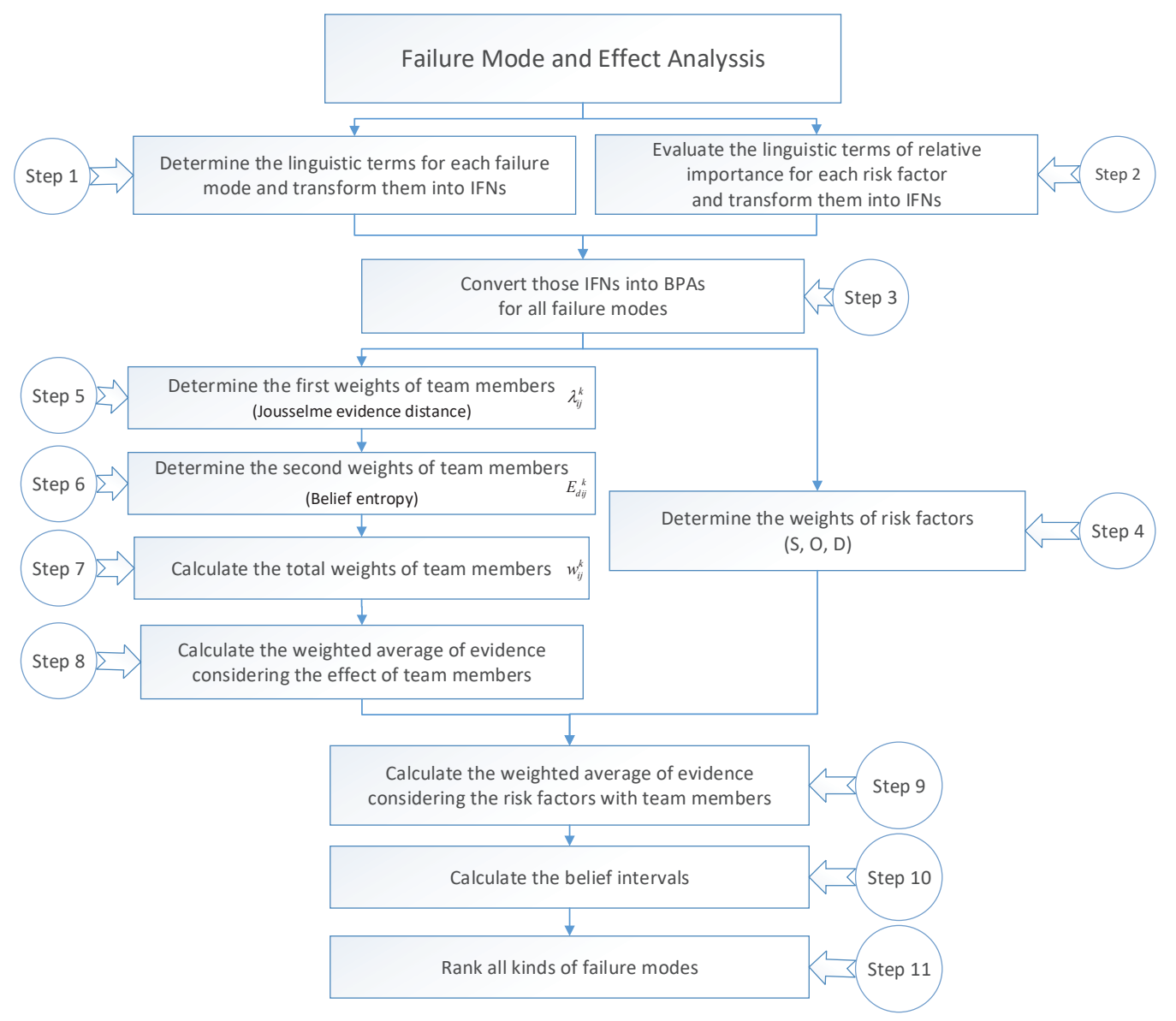

Figure 1. The flow chart of our proposed method.

Step 1: Determine the linguistic terms for each failure mode and transform them into IFNs. The specific judgement levels are divided into ten linguistic parts (see in Tables 1-3), which contains Very very low (VVL), Very Low (VL), Low (L), Medium low (ML), Medium (M), Medium high (MH), High (H), Very high $(\mathrm{VH})$, Very very high (VVH) and Extremely high (EH).

Table 1. The conversion and interpretation of the the relative importance evaluation under risk factor S.

\begin{tabular}{lll}
\hline The Linguistic Variables & IFNs & Severity (S) \\
\hline Very very low (VVL) & $(0.10,0.90)$ & Almost no casualties \\
Very low (VL) & $(0.10,0.75)$ & Very low level of injuries of people and amount of property damage \\
Low (L) & $(0.25,0.60)$ & Low level of injuries of people and amount of property damage \\
Medium low (ML) & $(0.40,0.50)$ & medium level of injuries of people and amount of property damage \\
Medium (M) & $(0.50,0.40)$ & moderate level of injuries of people and amount of property damage \\
Medium high (MH) & $(0.60,0.30)$ & moderately high level of injuries of people and amount of property damage \\
High (H) & $(0.70,0.20)$ & high level of injuries of people and amount of property damage \\
Very high (VH) & $(0.80,0.10)$ & Very high level of injuries of people and amount of property damage \\
Very very high (VVH) & $(0.90,0.10)$ & Very very high level of injuries of people and amount of property damage \\
Extremely high (EH) & $(1.00,0.00)$ & Severe level of injuries or death of people and amount of property damage \\
\hline
\end{tabular}


Table 2. The conversion and interpretation of the the relative importance evaluation under risk factor O.

\begin{tabular}{lll}
\hline The Linguistic Variables & IFNs & Occurrence (O) \\
\hline Very very low (VVL) & $(0.10,0.90)$ & A failure almost unlikely to occur \\
Very low (VL) & $(0.10,0.75)$ & A failure is likely to occur once, but unlikely to occur more frequently \\
Low (L) & $(0.25,0.60)$ & A failure occurs in low probabilities \\
Medium low (ML) & $(0.40,0.50)$ & A failure occurs in moderately low probabilities \\
Medium (M) & $(0.50,0.40)$ & A failure occurs in moderate probability \\
Medium high (MH) & $(0.60,0.30)$ & A failure occurs in moderately high probabilities \\
High (H) & $(0.70,0.20)$ & A failure occurs in high probabilities \\
Very high (VH) & $(0.80,0.10)$ & A failure occurs in very high probabilities \\
Very very high (VVH) & $(0.90,0.10)$ & A failure occurs in very very high probabilities \\
Extremely high (EH) & $(1.00,0.00)$ & A failure occurs in extremely high probabilities \\
\hline
\end{tabular}

Table 3. The conversion and interpretation of the the relative importance evaluation under risk factor D.

\begin{tabular}{lll}
\hline The Linguistic Variables & IFNs & Detection (D) \\
\hline Very very low (VVL) & $(0.10,0.90)$ & The detection of failure occurrence is completely certain \\
Very low (VL) & $(0.10,0.75)$ & The detection of failure occurrence is almost certain \\
Low (L) & $(0.25,0.60)$ & The failure occurrence is very likely to be detected \\
Medium low (ML) & $(0.40,0.50)$ & The failure occurrence is likely to be detected \\
Medium (M) & $(0.50,0.40)$ & A moderate likelihood to detect the failure occurrence \\
Medium high (MH) & $(0.60,0.30)$ & A moderately small likelihood to detect the failure occurrence \\
High (H) & $(0.70,0.20)$ & A small probability of detecting the failure occurrence \\
Very high (VH) & $(0.80,0.10)$ & A low likelihood to detect the failure occurrence \\
Very very high (VVH) & $(0.90,0.10)$ & A very low likelihood to detect the failure occurrence \\
Extremely high (EH) & $(1.00,0.00)$ & Almost impossible to detect failure occurrence \\
\hline
\end{tabular}

Step 2: Evaluate the linguistic terms of relative importance for each risk factor and transform them into IFNs. Similarly, the specific judgement levels are divided into five parts (see in Table 4), which contains Very important, Important, Medium, Unimportant and Very unimportant.

Table 4. The linguistic variables for the importance of risk factors.

\begin{tabular}{ll}
\hline The Linguistic Variables & IFNs \\
\hline Very important & $(0.90,0.10,0.00)$ \\
Important & $(0.75,0.20,0.05)$ \\
Medium & $(0.50,0.45,0.05)$ \\
Unimportant & $(0.35,0.60,0.05)$ \\
Very unimportant & $(0.10,0.90,0.00)$ \\
\hline
\end{tabular}

Step 3: Convert all IFNs into BPAs for all failure modes. In addition, the concrete form can be defined as follows:

$$
\begin{gathered}
m_{i j}^{k}(Y e s)=\mu_{i j}^{k} \\
m_{i j}^{k}(N o)=v_{i j}^{k} \\
m_{i j}^{k}(Y e s, N o)=1-\mu_{i j}^{k}-v_{i j}^{k} .
\end{gathered}
$$

Step 4: Determine the weights of risk factors. For the three judgement model S, O and D, each model can be transformed into an IFS to represent the information value. Based on the weight calculation, which is proposed by Boran et al. [16], the weight $w_{j}$ can be obtained. The computational equations are defined as follows: 


$$
w_{j}=\frac{\left(\mu_{j}+\pi_{j}\left(\mu_{j} /\left(\mu_{j}+v_{\mathrm{j}}\right)\right)\right)}{\sum_{j=1}^{n}\left(\mu_{j}+\pi_{j}\left(\mu_{j} /\left(\mu_{j}+v_{\mathrm{j}}\right)\right)\right)},
$$

which satisfy the condition that

$$
\sum_{j=1}^{n} w_{j}=1
$$

Step 5: Determine the weights of team members $\lambda_{i j}^{k}$ by using evidence distance which is introduced by Jousselme et al. [29].

Assume two groups of BPAs $m_{i j}^{q}(Y e s), m_{i j}^{q}(N o), m_{i j}^{q}(Y e s, N o)$ and $m_{i j}^{t}(Y e s), m_{i j}^{t}(N o), m_{i j}^{t}(Y e s, N o)$, $(q, t=1,2, \ldots, p)$ are two bodies of evidence (BOE) , obtained by two different team members. In this paper, the similarity function, using the evidence distance to define the distance $d\left(m_{i j}^{q}, m_{i j}^{t}\right)$ between $m_{i j}^{q}$ and $m_{i j}^{t}$, is proposed as follows:

$$
s\left(m_{i j}^{q}, m_{i j}^{t}\right)=1-d\left(m_{i j}^{q}, m_{i j}^{t}\right)
$$

The $\operatorname{Sup}\left(m_{i j}^{k}\right)$ is used to represent the degree of $m_{i j}^{k}$ supported by other bodies of evidence. In addition, the reliability degree $\mathrm{Crd}\left(m_{i j}^{k}\right)$ are defined as follows:

$$
\begin{gathered}
\operatorname{Crd}\left(m_{i j}^{k}\right)=\frac{\operatorname{Sup}\left(m_{i j}^{k}\right)}{\sum_{t=1}^{p} \operatorname{Sup}\left(m_{i j}^{t}\right)}, \\
\operatorname{Sup}\left(m_{i j}^{k}\right)=\sum_{t=1, t \neq k}^{p} s\left(m_{i j}^{k}, m_{i j}^{t}\right) .
\end{gathered}
$$

The $\operatorname{Crd}\left(m_{i j}^{k}\right)$ is to define the $\lambda_{i j}^{k}$

$$
\lambda_{i j}^{k}=\operatorname{Crd}\left(m_{i j}^{k}\right)=\frac{\operatorname{Sup}\left(m_{i j}^{k}\right)}{\sum_{t=1}^{p} \operatorname{Sup}\left(m_{i j}^{t}\right)} .
$$

Step 6: Determine the weights of team members $E_{d i j}^{k}$ by using belief entropy [26]. Based on the fellow steps, for all team members, their information value has been transformed into IFSs. Then, another weight $E_{d i j}^{k}$ is calculated, which expresses the amount of uncertainty for all propositions. The specific equation is defined as follows:

$$
\begin{aligned}
& E_{d i j}^{k}= \\
& -m_{i j}^{k}(Y) \log _{2} \frac{m_{i j}^{k}(Y)}{2^{|Y|}-1}-m_{i j}^{k}(N) \log _{2} \frac{m_{i j}^{k}(N)}{2^{|N|}-1}-m_{i j}^{k}(Y, N) \log _{2} \frac{m_{i j}^{k}(Y, N)}{2^{|Y, N|}-1} .
\end{aligned}
$$

Step 7: Calculate the total weights of team members $w_{i j}^{k}$ by combing $\lambda_{i j}^{k}$ and $E_{d i j}^{k}$. After obtaining the two weights, the total weights of FMEA team members can be calculated as the form of multiplication, which is defined as follows:

$$
\begin{gathered}
w_{i j}^{k}=\frac{\lambda_{i j}^{k} E_{d i j}^{k}}{\sum_{n=1}^{p} \lambda_{i j}^{k} E_{d i j}^{k}}, \\
\sum_{k=1}^{p} w_{i j}^{k}=1,
\end{gathered}
$$


where $p$ is the total number of failure modes for each risk factor.

Step 8: Calculate the weighted average of evidence considering the team members' effect of FMEA model. For each failure mode $F M_{i}$, there exists a group of basic probability assignment functions to express the degree of importance, which can be denoted as $m(Y e s) m(N o)$ and $m(Y e s, N o)$. Thus, after obtaining the $w_{i j}^{k}$ weights, the weighted average can be obtained as follows:

$$
\begin{gathered}
m_{i j}^{\prime \prime}(\text { Yes })=\sum_{k=1}^{p} w_{i j}^{k} m_{i j}^{k}(\text { Yes }), \\
m_{i j}^{\prime \prime}(N o)=\sum_{k=1}^{p} w_{i j}^{k} m_{i j}^{k}(N o), \\
m_{i j}^{\prime \prime}(\text { Yes, No })=1-\sum_{k=1}^{p} w_{i j}^{k} m_{i j}^{k}(Y e s)-\sum_{k=1}^{p} w_{i j}^{k} m_{i j}^{k}(N o) .
\end{gathered}
$$

Step 9: Calculate the weighted average of evidence considering the risk factors with team members. To consider the impact of different risk factors $(\mathrm{S}, \mathrm{O}, \mathrm{D})$, the weighted average of evidence which can be denoted as $m_{i}^{\prime}(Y e s), m_{i}^{\prime}(N o)$ and $m_{i}^{\prime}(Y e s, N o)$ is expressed as follows:

$$
\begin{gathered}
m_{i}^{\prime}(\text { Yes })=\sum_{j=1}^{n} w_{j} m_{i j}^{\prime \prime}(\text { Yes }), \\
m_{i}^{\prime}(\mathrm{No})=\sum_{j=1}^{n} w_{j} m_{i j}^{\prime \prime}(\mathrm{No}), \\
m_{i}^{\prime}(\text { Yes, No })=1-\sum_{j=1}^{n} w_{j} m_{i j}^{\prime \prime}(Y e s)-\sum_{j=1}^{n} w_{j} m_{i j}^{\prime \prime}(\mathrm{No}) .
\end{gathered}
$$

Step 10: Calculate the belief intervals. After obtaining the weighted average of evidence in Step 9, the belief interval $\left[\operatorname{Bel}\left(F M_{i}\right), \operatorname{Pl}\left(F M_{i}\right)\right]$ which is used to show the degree of support and opposition can be determined as:

$$
\begin{gathered}
\operatorname{Bel}\left(F M_{i}\right)=m_{i}^{\prime}(Y e s), \\
\operatorname{Pl}\left(F M_{i}\right)=m_{i}^{\prime}(Y e s)+m_{i}^{\prime}(Y e s, N o) .
\end{gathered}
$$

Step 11: Rank all kinds of failure modes. Based on the belief intervals, the risk of different failure model can be compared with others by using Equation (19) . After the process of comparison, the list of ranking in FMEA can be obtained.

\section{Application}

In this section, an example is used to illustrate the complete procedures of the proposed method.

The risk evaluation process has a great impact in many fields, such as multi-criteria decision-making (MCDM) [62-65] and other works [66,67]. In most situations, the weights for each risk factor may change the final result and lead the decision maker to make the undeserved judgement. To modify the process of products production as an easier and lower-cost method, the Failure mode and Effects Analysis play a growing important role in modern society.

Thus, an FMEA team consisting of five functional team members identifies potential failure modes in the electronics manufacturing project and wants to prioritize them in terms of their risk factors such as $\mathrm{S}$ (Severity), $\mathrm{O}$ (Occurrence) and $\mathrm{D}$ (Detection). In addition, twelve failure modes are identified. For the difficulty of evaluating the risk factors, the FMEA team members in this numerical example are supposed to assess them employing the linguistic terms. The specific transforming process is shown in Tables 1-3. 
Step 1: The assessment information of the twelve failure modes on each risk factor, which was provided by the five team members, can be illustrated in Table 5. Each team member comes from different department, such as manufacturing, engineering, design and technique. Considering their deferent specialities and functions, the weights are determined by their degree of importance.

Table 5. The linguistic evaluation for each failure mode.

\begin{tabular}{|c|c|c|c|c|c|c|c|c|c|c|c|c|c|c|c|}
\hline \multirow{2}{*}{ Failure Mode } & \multicolumn{5}{|c|}{$S$} & \multicolumn{5}{|c|}{ O } & \multicolumn{5}{|c|}{ D } \\
\hline & $\mathrm{TM}_{1}$ & $\mathrm{TM}_{2}$ & $\mathrm{TM}_{3}$ & $\mathrm{TM}_{4}$ & $\mathrm{TM}_{5}$ & $\mathrm{TM}_{1}$ & $\mathrm{TM}_{2}$ & $\mathrm{TM}_{3}$ & $\mathrm{TM}_{4}$ & $\mathrm{TM}_{5}$ & $\mathrm{TM}_{1}$ & $\mathrm{TM}_{2}$ & $\mathrm{TM}_{3}$ & $\mathrm{TM}_{4}$ & $\mathrm{TM}_{5}$ \\
\hline$F M_{1}$ & $\mathrm{VH}$ & VVH & $\mathrm{L}$ & $\mathrm{H}$ & $\mathrm{VH}$ & M & $\mathrm{MH}$ & ML & $\mathrm{H}$ & $\mathrm{MH}$ & ML & ML & $\mathrm{MH}$ & $M$ & $\mathrm{M}$ \\
\hline$F M_{2}$ & VVH & $\mathrm{VH}$ & $\mathrm{VH}$ & VVL & $\mathrm{VH}$ & M & M & $\mathrm{MH}$ & VL & M & ML & $\mathrm{MH}$ & $\mathrm{MH}$ & $\mathrm{H}$ & M \\
\hline$F M_{3}$ & $\mathrm{H}$ & $\mathrm{MH}$ & $\mathrm{VH}$ & $\mathrm{L}$ & $\mathrm{H}$ & ML & M & $\mathrm{L}$ & $\mathrm{MH}$ & M & ML & M & $\mathrm{L}$ & $\mathrm{L}$ & $\mathrm{MH}$ \\
\hline$F M_{4}$ & M & ML & $\mathrm{H}$ & M & MH & $\mathrm{H}$ & M & ML & $\mathrm{H}$ & M & ML & ML & $\mathrm{H}$ & $\mathrm{L}$ & $\mathrm{L}$ \\
\hline$F M_{5}$ & $\mathrm{~L}$ & M & $\mathrm{MH}$ & $\mathrm{MH}$ & M & $\mathrm{H}$ & ML & VVL & ML & $\mathrm{L}$ & $\mathrm{L}$ & $\mathrm{L}$ & ML & ML & M \\
\hline$F M_{6}$ & $\mathrm{~L}$ & ML & M & $\mathrm{L}$ & M & M & $\mathrm{MH}$ & $\mathrm{L}$ & M & $\mathrm{MH}$ & $\mathrm{MH}$ & ML & M & ML & ML \\
\hline$F M_{7}$ & M & $\mathrm{L}$ & $\mathrm{H}$ & M & $\mathrm{MH}$ & M & M & $\mathrm{L}$ & $\mathrm{H}$ & M & ML & M & $\mathrm{MH}$ & $\mathrm{M}$ & ML \\
\hline$F M_{8}$ & $\mathrm{M}$ & ML & $\mathrm{MH}$ & $\mathrm{L}$ & $\mathrm{H}$ & $\mathrm{L}$ & $\mathrm{MH}$ & $\mathrm{MH}$ & $\mathrm{L}$ & M & $\mathrm{H}$ & $\mathrm{MH}$ & $\mathrm{L}$ & $\mathrm{MH}$ & VL \\
\hline$F M_{9}$ & $\mathrm{~L}$ & M & $\mathrm{H}$ & $\mathrm{L}$ & M & M & $\mathrm{MH}$ & $\mathrm{L}$ & VL & $\mathrm{L}$ & $\mathrm{L}$ & $\mathrm{M}$ & $\mathrm{M}$ & ML & $\mathrm{L}$ \\
\hline$F M_{10}$ & $\mathrm{~L}$ & ML & ML & $\mathrm{M}$ & M & $\mathrm{L}$ & $\mathrm{L}$ & $\mathrm{L}$ & ML & M & ML & $\mathrm{MH}$ & ML & ML & ML \\
\hline$F M_{11}$ & $\mathrm{~L}$ & M & $\mathrm{MH}$ & M & M & $\mathrm{L}$ & $\mathrm{M}$ & $\mathrm{M}$ & $\mathrm{M}$ & M & $\mathrm{L}$ & M & M & M & M \\
\hline$F M_{12}$ & ML & M & ML & $\mathrm{L}$ & $\mathrm{MH}$ & $\mathrm{M}$ & VL & VL & VL & VL & ML & $\mathrm{L}$ & $\mathrm{M}$ & VL & $\mathrm{L}$ \\
\hline
\end{tabular}

Step 2: Evaluate the linguistic terms of relative importance for each risk factor and transform them into IFNs (see in Table 6).

Table 6. The importance of risk factors.

\begin{tabular}{ccc}
\hline Risk Factors & Mode Abbreviation & The Linguistic Variables \\
\hline Severity & $\mathrm{S}$ & Very important \\
Occurrence & $\mathrm{O}$ & Important \\
Detection & $\mathrm{D}$ & Medium \\
\hline
\end{tabular}

Step 3: Convert those IFNs into BPAs for all failure modes. The specific transforming equation is shown in Equations (12)-(14).

Step 4: Determine the weights of risk factors. In this paper, the weights calculation of risk factors in this example is shown in Equations (27) and (28). In addition, the results are shown in Table 7.

Table 7. The weights of risk factors.

\begin{tabular}{cc}
\hline Risk Factors & Weights \\
\hline Severity & 0.4009 \\
Occurrence & 0.3516 \\
Detection & 0.2475 \\
\hline
\end{tabular}

Step 5: Determine the first weights of team members $\lambda_{i j}^{k}$ by using evidence distance [29]. The specific value of each mode is shown in Table 8. 
Table 8. The $\lambda_{i j}^{k}$ weights for each failure mode.

\begin{tabular}{|c|c|c|c|c|c|c|c|c|c|c|c|c|c|c|c|}
\hline \multirow{2}{*}{ Failure Mode } & \multicolumn{5}{|c|}{ Severity (S) } & \multicolumn{5}{|c|}{ Occurrence $(\mathrm{O})$} & \multicolumn{5}{|c|}{ Detection (D) } \\
\hline & $\mathrm{TM}_{1}$ & $\mathrm{TM}_{2}$ & $\mathrm{TM}_{3}$ & $\mathbf{T M}_{4}$ & $\mathbf{T M}_{5}$ & $\mathrm{TM}_{1}$ & $\mathbf{T M}_{2}$ & $\mathbf{T M}_{3}$ & $\mathbf{T M}_{4}$ & $\mathrm{TM}_{5}$ & $\mathrm{TM}_{1}$ & $\mathrm{TM}_{2}$ & $\mathrm{TM}_{3}$ & $\mathrm{TM}_{4}$ & $\mathrm{TM}_{5}$ \\
\hline$F M_{1}$ & 0.2118 & 0.2117 & 0.1463 & 0.2184 & 0.2118 & 0.2035 & 0.2093 & 0.1860 & 0.1919 & 0.2093 & 0.2000 & 0.2000 & 0.1889 & 0.2056 & 0.2056 \\
\hline$F$ & & & & & 0.2299 & & 0.2157 & & & 0.2 & & 0.2093 & 0.2093 & & 0.2035 \\
\hline$F M_{3}$ & 172 & 0.2248 & 0.1939 & & & & & & & & & 0.2032 & 13 & 0.2013 & 0.1851 \\
\hline$F M_{4}$ & 093 & 0.19 & 0.1860 & 0.2 & 0.2035 & 0.2 & 0.2056 & 0.1 & 0.2 & 0.2 & & 0.2101 & 0.1 & 0.2025 & 0.2025 \\
\hline$F M_{5}$ & 700 & 0.21 & 0.2 & & 0.21 & 0.1 & 0.2268 & 0. & & 0.2 & & 0.198 & 0. & 0.2 & 0.1896 \\
\hline$F M_{6}$ & & & & & & & 0.2 & & & & & 0.2056 & & 0 . & 0.2056 \\
\hline$F M_{7}$ & & & & & 0.20 & & 0.20 & & & & & 0.2056 & & & 0.2000 \\
\hline$F M_{8}$ & & 0.2 & & & & & 0.2020 & & & & & 0.2199 & & & 0.1648 \\
\hline$F M_{9}$ & & & & & 0.21 & & 0.18 & & & 0.2 & & 0.199 & & & 0.1978 \\
\hline$F M_{10}$ & 846 & 0.2066 & 0.2066 & 0.2011 & 0.20 & 0.2099 & 0.2099 & 0.2 & 0.1 & 0.2 & 0.2 & 0.1739 & 0.2 & 0.2 & 0.2065 \\
\hline$F M_{11}$ & 0.1718 & 0.2113 & 0.1941 & 0.2113 & 0.2113 & 0.1697 & 0.2076 & 0.2076 & 0.2076 & 0.2076 & & 0.2092 & 0.1 & 0.2 & 0.1772 \\
\hline$F M_{12}$ & 0.2104 & 0.2047 & 0.2104 & 0.1874 & 0.1871 & 0.1466 & 0.2133 & 0.2133 & 0.2133 & 0.2133 & 0.2063 & 0.1989 & 0.1896 & 0.2063 & 0.1989 \\
\hline
\end{tabular}

Step 6: Determine the second weights of team members $E_{d i j}^{k}$ by using belief entropy. In addition, the results are shown in Table 9.

Table 9. The $E_{d i j}^{k}$ weights for each failure mode.

\begin{tabular}{|c|c|c|c|c|c|c|c|c|c|c|c|c|c|c|c|}
\hline \multirow{2}{*}{ Failure Mode } & \multicolumn{5}{|c|}{ Severity (S) } & \multicolumn{5}{|c|}{ Occurrence (O) } & \multicolumn{5}{|c|}{ Detection (D) } \\
\hline & $\mathrm{TM}_{1}$ & $\mathrm{TM}_{2}$ & $\mathrm{TM}_{3}$ & $\mathrm{TM}_{4}$ & $\overline{\mathrm{TM}_{5}}$ & $\mathrm{TM}_{1}$ & $\mathrm{TM}_{2}$ & $\mathrm{TM}_{3}$ & $\mathrm{TM}_{4}$ & $\overline{T_{M}}$ & $\mathrm{TM}_{1}$ & $\mathrm{TM}_{2}$ & $\mathrm{TM}_{3}$ & $\mathrm{TM}_{4}$ & $\overline{\mathrm{TM}_{5}}$ \\
\hline$F M_{1}$ & 1.0804 & 0.4690 & 1.5904 & 1.3153 & 1.0804 & 1.5195 & 1.4540 & 1.5195 & 1.3153 & 1.4540 & 1.5195 & 1.5195 & 1.4540 & 1.5195 & 1.519 \\
\hline$F M_{2}$ & 0 & & & & 153 & & & & & & & 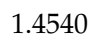 & & & 195 \\
\hline$F M_{3}$ & 153 & 1.4540 & $1.0 \varepsilon$ & & 1.3 & 1.5 & & 1.5 & & 1.5195 & 95 & 1.5195 & 1.5905 & 1.5905 & 1.4540 \\
\hline$F M_{4}$ & 1.5195 & 1.5 & 1.3 & 1.5 & 1.4 & & & 1. & & 1.5 & 1. & 1.5195 & 1.3153 & 1. & 1.5905 \\
\hline$F M_{5}$ & 905 & 1.5 & 1.4 & & 1.5 & & 1. & 0 & & 1. & & 1. & 1. & 1. & 1.5195 \\
\hline$F M_{6}$ & & 1.5 & & & 1.5 & & 1. & & & & & 1. & 1 & 1 & 1.5 \\
\hline$F M_{7}$ & & & & & & & & & & & & & & 1. & 1.5195 \\
\hline$F M_{8}$ & & & & & & & & & & & & & & & 1.2918 \\
\hline$F M_{9}$ & & & & & & & & & & & & & & & 1.5905 \\
\hline$F M_{10}$ & & & & & & & & & & & & & & & \\
\hline$F N$ & & & & & & & & & & & & & & & 1.4540 \\
\hline$F M_{12}$ & 1.5195 & 1.5195 & 1.5195 & 1.5905 & 1.4540 & 1.5195 & 1.2918 & 1.2918 & 1.2918 & 1.2918 & 1.5195 & 1.5905 & 1.5195 & 1.2918 & 1.5905 \\
\hline
\end{tabular}

Step 7: Calculate the total weights of team members $w_{i j}^{k}$ by combing $\lambda_{i j}^{k}$ and $E_{d i j}^{k}$. After the process of normalization, the specific value of weights is shown in Table 10.

Table 10. The total weights $w_{i j}^{k}$ of team members for each failure mode.

\begin{tabular}{|c|c|c|c|c|c|c|c|c|c|c|c|c|c|c|c|}
\hline \multirow{2}{*}{ Failure Mode } & \multicolumn{5}{|c|}{ Severity (S) } & \multicolumn{5}{|c|}{ Occurrence $(\mathrm{O})$} & \multicolumn{5}{|c|}{ Detection (D) } \\
\hline & $\mathbf{T M}_{1}$ & $\mathrm{TM}_{2}$ & $\mathrm{TM}_{3}$ & $\mathbf{T M}_{4}$ & $\mathrm{TM}_{5}$ & $\mathrm{TM}_{1}$ & $\mathrm{TM}_{2}$ & $\mathrm{TM}_{3}$ & $\mathrm{TM}_{4}$ & $\mathrm{TM}_{5}$ & $\mathrm{TM}_{1}$ & $\mathbf{T M}_{2}$ & $\mathrm{TM}_{3}$ & $\mathrm{TM}_{4}$ & $\mathrm{TM}_{5}$ \\
\hline$F M_{1}$ & 0.2125 & 0.0922 & 0.2161 & 0.2667 & 0.2125 & 0.2128 & 0.2095 & 0.1945 & 0.1737 & 0.2095 & 0.2016 & 0.2016 & 0.182 & 0.2073 & 0.207 \\
\hline$I_{2}$ & 0.1078 & 0.2675 & 0.2675 & 0.0394 & 0.3178 & 0.2229 & 0.2229 & 0.1885 & 0.1428 & 0.2229 & & 0.2095 & 0.2095 & 0.1737 & 0.2128 \\
\hline$F M_{3}$ & 0.2107 & 0.2411 & 0.1545 & 0.2339 & 0.1597 & 0. & 0.2105 & 0.1902 & 0.1 & 0.20 & 67 & 0.2010 & 0.2 & 0.2085 & 0.1753 \\
\hline$F M_{4}$ & 0.2166 & 0.1986 & 0.16 & & 0.20 & & 0.2172 & 0.17 & 0.1 & 0.21 & 10 & 0.2110 & 0.1 & 0.2129 & 0.2129 \\
\hline$F M_{5}$ & 0.1797 & 0.2125 & 0.19 & 0.1977 & 0.212 & & 0.25 & 0.0591 & 0.2 & 0.25 & 29 & 0.21 & 0.2010 & 0.2010 & 0.1848 \\
\hline$F M_{6}$ & 033 & 0.2014 & 0.19 & 0.2033 & 0.19 & 0.2 & 0.21 & 0.1 & 0.2 & 0.2 & 0 . & 0.207 & 0.2 & 0 & 0.2072 \\
\hline$F M_{7}$ & & & & & 0. & & 0.2 & & & 0.2 & & 0.209 & 0.2 & 0. & 0.2035 \\
\hline$F M_{8}$ & & & & & & & & & & & & 0.2242 & & & 0.1492 \\
\hline FM & & 0.21 & & & 0.21 & & 0.1762 & & & 0.22 & & 0.1961 & & & $=0.20$ \\
\hline$F M_{10}$ & 916 & 0.2048 & 0.2048 & 0.1993 & 0.1993 & 0.2134 & 0.2134 & 0.2134 & 0.1640 & 0.19 & 0.2081 & 0.1677 & 0.2081 & 0.2081 & 0.2081 \\
\hline$F M$ & & 0.2114 & 0.1858 & 0.2114 & 0.2114 & & 0.2059 & & 0.2059 & 0.2059 & & 0.2059 & 0.2059 & 0.2059 & 0.2059 \\
\hline$F M_{12}$ & 0.2103 & 0.2046 & 0.2103 & 0.1960 & 0.1789 & 0.1681 & 0.2080 & 0.2080 & 0.2080 & 0.2080 & 0.2089 & 0.2108 & 0.1920 & 0.1776 & 0.2108 \\
\hline
\end{tabular}

Step 8: Calculate the weighted average of evidence considering the team members' effect of FMEA model. The results are shown in Table 11. 
Table 11. The weighted average of team members.

\begin{tabular}{|c|c|c|c|c|c|c|c|c|c|}
\hline \multirow{2}{*}{ Failure Mode } & \multicolumn{3}{|c|}{ Severity (S) } & \multicolumn{3}{|c|}{ Occurrence $(\mathrm{O})$} & \multicolumn{3}{|c|}{ Detection (D) } \\
\hline & $m_{i j}^{\prime \prime}(Y e s)$ & $m_{i j}^{\prime \prime}(N o)$ & $m_{i j}^{\prime \prime}($ Yes, No $)$ & $m_{i j}^{\prime \prime}(Y e s)$ & $m_{i j}^{\prime \prime}(\mathrm{No})$ & $m_{i j}^{\prime \prime}($ Yes, No $)$ & $m_{i j}^{\prime \prime}(Y e s)$ & $m_{i j}^{\prime \prime}(N o)$ & $m_{i j}^{\prime \prime}(Y e s, N o)$ \\
\hline$F M_{1}$ & 0.6637 & 0.2347 & 0.1015 & 0.5572 & 0.3428 & 0.1000 & 0.4779 & 0.4221 & 0.1000 \\
\hline$F M_{2}$ & 0.7514 & 0.1633 & 0.0853 & 0.4806 & 0.4123 & 0.1071 & 0.5572 & 0.3428 & 0.1000 \\
\hline$F M_{3}$ & 0.5867 & 0.3022 & 0.1116 & 0.4505 & 0.4400 & 0.1095 & 0.3926 & 0.4865 & 0.1208 \\
\hline $\mathrm{FM}_{4}$ & 0.5336 & 0.3663 & 0.0100 & 0.5532 & 0.3467 & 0.1000 & 0.3817 & 0.4970 & 0.1213 \\
\hline$F M_{5}$ & 0.4946 & 0.3964 & 0.1090 & 0.3922 & 0.5007 & 0.1070 & 0.3565 & 0.5018 & 0.1417 \\
\hline$F M_{6}$ & 0.3782 & 0.5014 & 0.1203 & 0.5139 & 0.3803 & 0.1057 & 0.4555 & 0.4444 & 0.1000 \\
\hline$F M_{7}$ & 0.5080 & 0.3828 & 0.1093 & 0.4805 & 0.4086 & 0.1109 & 0.4779 & 0.4221 & 0.1000 \\
\hline$F M_{8}$ & 0.4836 & 0.4065 & 0.1098 & 0.4372 & 0.4425 & 0.1203 & 0.4670 & 0.4146 & 0.1184 \\
\hline$F M_{9}$ & 0.4259 & 0.4532 & 0.1209 & 0.3385 & 0.5305 & 0.1310 & 0.3782 & 0.5014 & 0.1203 \\
\hline$F M_{10}$ & 0.4111 & 0.4793 & 0.1096 & 0.3236 & 0.5444 & 0.1320 & 0.4335 & 0.4665 & 0.1000 \\
\hline$F M_{11}$ & 0.4736 & 0.4714 & 0.1090 & 0.4560 & 0.4352 & 0.1088 & 0.4560 & 0.4352 & 0.1088 \\
\hline$F M_{12}$ & 0.4268 & 0.4634 & 0.1098 & 0.1672 & 0.6911 & 0.1416 & 0.3027 & 0.5673 & 0.1299 \\
\hline
\end{tabular}

Step 9: Calculate the weighted average of evidence considering the risk factors with team members. By introducing the consideration of risk factors, the weighted average of evidence is calculated. In addition, the results are shown in Table 12.

Step 10: Calculate the belief intervals. With the Equations (41) and (42), the final results are also shown in Table 12.

Table 12. The weighted average of evidence considering the risk factors with team members and the belief intervals.

\begin{tabular}{ccccccc}
\hline Failure Mode & $\mathbf{m}_{\mathbf{i j}}^{\prime}($ Yes $)$ & $\mathbf{m}_{\mathbf{i j}}^{\prime}(\mathbf{N o})$ & $\mathbf{m}_{\mathbf{i j}}^{\prime}($ Yes, No $)$ & Bel & P1 & Ranking \\
\hline$F M_{1}$ & 0.5932 & 0.3060 & 0.1007 & 0.5932 & 0.6939 & 2 \\
$F M_{2}$ & 0.6081 & 0.2953 & 0.0966 & 0.6081 & 0.7047 & 1 \\
$F M_{3}$ & 0.5037 & 0.3842 & 0.1121 & 0.5037 & 0.6158 & 4 \\
$F M_{4}$ & 0.5160 & 0.3804 & 0.1035 & 0.5160 & 0.6195 & 3 \\
$F M_{5}$ & 0.4335 & 0.4529 & 0.1136 & 0.4335 & 0.5471 & 9 \\
$F M_{6}$ & 0.4418 & 0.4466 & 0.1116 & 0.4418 & 0.5534 & 8 \\
$F M_{7}$ & 0.4927 & 0.3989 & 0.1083 & 0.4927 & 0.6010 & 5 \\
$F M_{8}$ & 0.4634 & 0.4213 & 0.1152 & 0.4634 & 0.5786 & 6 \\
$F M_{9}$ & 0.3853 & 0.4901 & 0.1246 & 0.3853 & 0.5099 & 10 \\
$F M_{10}$ & 0.3859 & 0.4990 & 0.1151 & 0.3859 & 0.5010 & 11 \\
$F M_{11}$ & 0.4476 & 0.4396 & 0.1127 & 0.4476 & 0.5603 & 7 \\
$F M_{12}$ & 0.3090 & 0.5659 & 0.1251 & 0.3090 & 0.4351 & 12 \\
\hline
\end{tabular}

Step 11: Rank all kinds of failure modes. After the process of comparison, the final ranking can be obtained (see in Table 13).

Table 13. The comparison of final ranking in different methods.

\begin{tabular}{ccccc}
\hline Failure Mode & Method 1 & Method 2 & Method 3 & Proposed Method \\
\hline$F M_{1}$ & 2 & 2 & 2 & 2 \\
$F M_{2}$ & 1 & 1 & 1 & 1 \\
$F M_{3}$ & 5 & 6 & 6 & 4 \\
$F M_{4}$ & 4 & 4 & 4 & 3 \\
$F M_{5}$ & 9 & 11 & 9 & 9 \\
$F M_{6}$ & 8 & 7 & 8 & 8 \\
$F M_{7}$ & 3 & 5 & 3 & 5 \\
$F M_{8}$ & 6 & 3 & 5 & 6 \\
\hline
\end{tabular}


Table 13. Cont.

\begin{tabular}{ccccc}
\hline Failure Mode & Method 1 & Method 2 & Method 3 & Proposed Method \\
\hline$F M_{9}$ & 10 & 9 & 11 & 10 \\
$F M_{10}$ & 11 & 10 & 10 & 11 \\
$F M_{11}$ & 7 & 8 & 7 & 7 \\
$F M_{12}$ & 12 & 12 & 12 & 12 \\
\hline
\end{tabular}

Here, we present some discussion about the proposed method.

In the previous related research, many scholars have tried to enhance the effectiveness and availability based on intuitionistic fuzzy sets, evidence theory and so on. The ranking comparisons of all the related works are shown in Table 13. There are some ranking differences among those methods. In general, the higher ranked models are $F M_{1}, F M_{2}$, and the lowest ranked model is $F M_{12}$, which is consistent with the previous three methods. For other failure modes, it can be seen that, in the evaluation of the proposed method, the overall ordering of $F M_{3}$ and $F M_{4}$ is slightly higher than the previous method, while the remaining rankings are generally consistent. The main reasons are summarized as follows:

The relatively importance of team members are different. In the method proposed by Liu et al. [15], the relative weights were supposed in advance, which are $0.10,0.15,0.20,0.25$ and 0.30 . In addition, in the intuitionistic fuzzy TOPSIS method, the impacts of team members are not considered. In addition, the method proposed by Guo [17] has just considered the conflict of team members simply. In our proposed method, the weights of team members are defined by using both the evidence distance [29] and the belief entropy [26]. The evidence distance is to show the degree of conflict for all team members. In addition, the belief entropy is used to reflect the uncertainty of the information of each team member. The combination of them can express the evaluated information completely and effectively. To be specific, since the uncertainty information contained in the results of the expert evaluations in $F M_{1}$, $F M_{2}$ and $F M_{12}$ is relatively low in this application, the weights obtained by considering the entropy factor has little effect on the final evaluation. Moreover, in $F M_{3}$ and $F M_{4}$, the overall uncertainty of the expert evaluation is relatively high, the second weights obtained by calculating the belief entropy have a relatively large influence on the overall evaluation result, which leads to the final result as shown in Table 13.

Thus, with the differences mentioned above, the aggregation approaches for all kinds of methods are different. As a comparison, the process of our proposed method to determine the weights of team members is particularly scientific and effective, with strong practical significance and good performance.

\section{Conclusions}

FMEA has been regarded as an effective analysis approach to identify and rank the potential failure modes in many applications. However, the uncertainty of the experts' decision process is not taken into consideration, which is regarded as an essential factor in decision-making. In this paper, the impact of experts factor uncertainty is modelled. A hybrid method to determine the weights of team members is proposed based on the belief entropy. The application in FMEA illustrates the efficiency of the proposed method.

Author Contributions: Methodology, F.X.; Writing of the original draft, Z.L.

Funding: The authors greatly appreciate the reviews' suggestions and the editor's encouragement. This research is supported by the Chongqing Overseas Scholars Innovation Program (No. cx2018077).

Conflicts of Interest: The authors declare no conflicts of interest. 


\section{References}

1. Bian, T.; Zheng, H.; Yin, L.; Deng, Y. Failure mode and effects analysis based on D numbers and TOPSIS. Qual. Reliab. Eng. Int. 2018, 34, 501-515. [CrossRef]

2. Jiang, W.; Xie, C.; Zhuang, M.; Tang, Y. Failure mode and effects analysis based on a novel fuzzy evidential method. Appl. Soft Comput. 2017, 57, 672-683. [CrossRef]

3. Liu, H.; Liu, L.; Li, P. Failure mode and effects analysis using intuitionistic fuzzy hybrid weighted euclidean distance operator. Int. J. Syst. Sci. 2014, 45, 2012-2030. [CrossRef]

4. Liu, H.; You, J.; Shan, M.; Shao, L. Failure mode and effects analysis using intuitionistic fuzzy hybrid TOPSIS approach. Soft Comput. 2015, 19, 1085-1098. [CrossRef]

5. Li, Z.; Chen, L. A novel evidential FMEA method by integrating fuzzy belief structure and grey relational projection method. Eng. Appl. Artif. Intell. 2019, 77, 136-147. [CrossRef]

6. Chen, L.; Deng, Y. A new failure mode and effects analysis model using Dempster-Shafer evidence theory and grey relational projection method. Eng. Appl. Artif. Intell. 2018, 76, 13-20. [CrossRef]

7. Wang, Z.; You, J.; Liu, H.; Wu, S. Failure mode and effect analysis using soft set theory and COPRAS method. Int. J. Comput. Intell. Syst. 2017, 10, 1002-1015. [CrossRef]

8. Sun, R.; Deng, Y. A new method to identify incomplete frame of discernment in evidence theory. IEEE Access 2019, 7, 15547-15555. [CrossRef]

9. Han, Y.; Deng, Y. An enhanced fuzzy evidential DEMATEL method with its application to identify critical success factors. Soft Comput. 2018, 22, 5073-5090. [CrossRef]

10. Liu, H.; You, J.; Fan, X.; Lin, Q. Failure mode and effects analysis using D numbers and grey relational projection method. Expert Syst. Appl. 2014, 41, 4670-4679. [CrossRef]

11. Tooranloo, H.S.; Ayatollah, A.S. A model for failure mode and effects analysis based on intuitionistic fuzzy approach. Appl. Soft Comput. 2016, 49, 238-247. [CrossRef]

12. Tooranloo, H.S.; Ayatollah, A.S.; Alboghobish, S. Evaluating knowledge management failure factors using intuitionistic fuzzy FMEA approach. Knowl. Inf. Syst. 2018, 57, 183-205. [CrossRef]

13. Seiti, H.; Hafezalkotob, A.; Najafi, S.E.; Khalaj, M. A risk-based fuzzy evidential framework for FMEA analysis under uncertainty: An interval-valued DS approach. J. Intell. Fuzzy Syst. 2018, 35, 1419-1430. [CrossRef]

14. Wang, Y.; Lei, Y.J. A technique for constructing intuitionistic fuzzy entropy. Control Decis. 2007, 22, 1390-1394.

15. Liu, H.-C.; Liu, L.; Liu, N. Risk evaluation approaches in failure mode and effects analysis: A literature review. Expert Syst. Appl. 2013, 40, 828-838. [CrossRef]

16. Boran, F.E.; Genç, S.; Kurt, M.; Akay, D. A multi-criteria intuitionistic fuzzy group decision-making for supplier selection with TOPSIS method. Expert Syst. Appl. 2009, 36, 11363-11368. [CrossRef]

17. Guo, J. A risk assessment approach for failure mode and effects analysis based on intuitionistic fuzzy sets and evidence theory. J. Intell. Fuzzy Syst. 2016, 30, 869-881. [CrossRef]

18. Chang, K.; Cheng, C. A risk assessment methodology using intuitionistic fuzzy set in FMEA. Int. J. Syst. Sci. 2010, 41, 1457-1471. [CrossRef]

19. Tzafestas, S.G. From the founding editor, dr. spyros g. tzafestas-30 th year anniversary of JINT. J. Intell. Rob. Syst. 2018, 91, 3-4. [CrossRef]

20. Tzafestas, S.G. Mobile robot control and navigation: A global overview. J. Intell. Rob. Syst. 2018, 91, 35-58. [CrossRef]

21. Fu, C.; Xu, D.-L.; Yang, S.-L. Distributed preference relations for multiple attribute decision analysis. J. Oper. Res. Soc. 2016, 67, 457-473. [CrossRef]

22. Jiang, W.; Wei, B. Intuitionistic fuzzy evidential power aggregation operator and its application in multiple criteria decision-making. Int. J. Syst. Sci. 2018, 49, 582-594. [CrossRef]

23. Wei, G.-W. GRA method for multiple attribute decision-making with incomplete weight information in intuitionistic fuzzy setting. Knowl.-Based Syst. 2010, 23, 243-247. [CrossRef]

24. Fei, L.; Wang, H.; Chen, L.; Deng, Y. A new vector valued similarity measure for intuitionistic fuzzy sets based on owa operators. Iran. J. Fuzzy Syst. 2018. [CrossRef]

25. Shannon, C.E. A mathematical theory of communication. Bell Syst. Tech. J. 1948, 27, 379-423. [CrossRef]

26. Deng, Y. Deng entropy. Chaos Solitons Fractals 2016, 91, 549-553. [CrossRef] 
27. Li, Y.; Deng, Y. Generalized ordered propositions fusion based on belief entropy. Int. J. Comput. Commun. Control 2018, 13, 792-807. [CrossRef]

28. Xiao, F. Multi-sensor data fusion based on the belief divergence measure of evidence and the belief entropy. Inf. Fusion 2019, 46, 23-32. [CrossRef]

29. Jousselme, A.; Maupin, P. Distances in evidence theory: Comprehensive survey and generalizations. Int. J. Approx. Reason. 2012, 53, 118-145. [CrossRef]

30. Dempster, A. Upper and lower probabilities induced by a multivalued mapping. Ann. Math. Stat. 1967, 38, 325-339. [CrossRef]

31. Xiao, F.; Zhan, C.; Lai, H.; Tao, L.; Qu, Z. New parallel processing strategies in complex event processing systems with data streams. Int. J. Distrib. Sens. Netw. 2017, 13, 1-15. [CrossRef]

32. Xiao, F. A novel multi-criteria decision-making method for assessing health-care waste treatment technologies based on D numbers. Eng. Appl. Artif. Intell. 2018, 71, 216-225. [CrossRef]

33. Fei, L.; Deng, Y.; Hu, Y. DS-VIKOR: A New Multi-criteria Decision-Making Method for Supplier Selection. Int. J. Fuzzy Syst. 2018. [CrossRef]

34. Chhipi-Shrestha, G.; Mori, J.; Hewage, K.; Sadiq, R. Clostridium difficile infection incidence prediction in hospitals (CDIIPH): A predictive model based on decision tree and fuzzy techniques. Stoch. Environ. Res. Risk Assess. 2017, 31, 417-430. [CrossRef]

35. Xiao, F. An intelligent complex event processing with D numbers under fuzzy environment. Math. Probl. Eng. 2016, 2016, 1-10.

36. Bian, T.; Deng, Y. Identifying influential nodes in complex networks: A node information dimension approach. Chaos 2018, 28. [CrossRef]

37. Zhang, H.; Deng, Y. Engine fault diagnosis based on sensor data fusion considering information quality and evidence theory. Adv. Mech. Eng. 2018, 10. [CrossRef]

38. Xiao, F. An Improved Method for Combining Conflicting Evidences Based on the Similarity Measure and Belief Function Entropy. Int. J. Fuzzy Syst. 2018, 20, 1256-1266. [CrossRef]

39. Xiao, F. A multiple criteria decision-making method based on D numbers and belief entropy. Int. J. Fuzzy Syst. 2019, accepted.

40. Gong, Y.; Su, X.; Qian, H.; Yang, N. Research on fault diagnosis methods for the reactor coolant system of nuclear power plant based on D-S evidence theory. Ann. Nucl. Energy 2018, 395-399. [CrossRef]

41. Michail, K.; Deliparaschos, K.M.; Tzafestas, S.G.; Zolotas, A.C. Ai-based actuator/sensor fault detection with low computational cost for industrial applications. IEEE Trans. Control Syst. Technol. 2016, 24, $293-301$. [CrossRef]

42. Cao, Z.; Lai, K.-L.; Lin, C.-T.; Chuang, C.-H.; Chou, C.-C.; Wang, S.-J. Exploring resting-state EEG complexity before migraine attacks. Cephalalgia 2018, 38, 1296-1306. [CrossRef] [PubMed]

43. Lin, C.T.; Chuang, C.H.; Cao, Z.; Singh, A.K.; Hung, C.S.; Yu, Y.H. Nascimben, M.; Liu, Y.T.; King, J.T.; $\mathrm{Su}, \mathrm{T}$.P.; et al. Forehead EEG in support of future feasible personal healthcare solutions: Sleep management, headache prevention, and depression treatment. IEEE Access 2017, 5, 10612-10621. [CrossRef]

44. Mo, H.; Deng, Y. A new MADA methodology based on D numbers. Int. J. Fuzzy Syst. 2018, 20, $2458-2469$. [CrossRef]

45. Fei, L.; Deng, Y. A new divergence measure for basic probability assignment and its applications in extremely uncertain environments. Int. J. Intell. Syst. 2018. [CrossRef]

46. Han, Y.; Deng, Y. A novel matrix game with payoffs of Maxitive Belief Structure. Int. J. Intell. Syst. 2018. [CrossRef]

47. Yin, L.; Deng, X.; Deng, Y. The negation of a basic probability assignment. IEEE Trans. Fuzzy Syst. 2019, 27, 135-143. [CrossRef]

48. Shafer, G. A Mathematical Theory of Evidence; Princeton University Press: Princeton, NJ, USA, 1976.

49. Dempster, A.P. Upper and lower probabilities induced by a multivalued mapping. In Classic Works of the Dempster-Shafer Theory of Belief Functions; Springer: Berlin, Germany, 2008; pp. 57-72.

50. Xu, H.; Deng, Y. Dependent evidence combination based on shearman coefficient and pearson coefficient. IEEE Access 2018, 6, 11634-11640. [CrossRef]

51. Zhang, W.; Deng, Y. Combining conflicting evidence using the DEMATEL method. Soft Comput. 2018. [CrossRef] 
52. Wang, Y.; Deng, Y. Base belief function: An efficient method of conflict management. J. Ambient Intell. Hum. Comput. 2018. [CrossRef]

53. Jiang, W.; Wei, B.; Liu, X.; Li, X.; Zheng, H. Intuitionistic fuzzy power aggregation operator based on entropy and its application in decision-making. Int. J. Intell. Syst. 2018, 33, 49-67. [CrossRef]

54. Wei, G.-W. Maximizing deviation method for multiple attribute decision-making in intuitionistic fuzzy setting. Knowl.-Based Syst. 2008, 21, 833-836. [CrossRef]

55. Atanassov, K.T. Intuitionistic fuzzy sets. Fuzzy Sets Syst. 1986, 20, 87-96. [CrossRef]

56. Dymova, L.; Sevastjanov, P. An interpretation of intuitionistic fuzzy sets in terms of evidence theory: Decision making aspect. Knowl.-Based Syst. 2010, 23, 772-782. [CrossRef]

57. Dymova, L.; Sevastjanov, P. The operations on interval-valued intuitionistic fuzzy values in the framework of Dempster-Shafer theory. Inf. Sci. 2016, 360, 256-272. [CrossRef]

58. Lin, J. Divergence measures based on the shannon entropy. IEEE Trans. Inf. Theory 1991, 37, $145-151$. [CrossRef]

59. Pan, L.; Deng, Y. A New Belief Entropy to Measure Uncertainty of Basic Probability Assignments Based on Belief Function and Plausibility Function. Entropy 2018, 20, 842. [CrossRef]

60. Kang, B.; Deng, Y.; Hewage, K.; Sadiq, R. Generating Z- number based on OWA weights using maximum entropy. Int. J. Intell. Syst. 2018, 33, 1745-1755. [CrossRef]

61. Xu, Z.; Yager, R.R. Some geometric aggregation operators based on intuitionistic fuzzy sets. Int. J. Gen. Syst. 2006, 35, 417-433. [CrossRef]

62. Kabir, G.; Sadiq, R.; Tesfamariam, S. A review of multi-criteria decision-making methods for infrastructure management. Struct. Infrastruct. Eng. 2014, 10, 1176-1210. [CrossRef]

63. Khan, F.; Sadiq, R.; Veitch, B. Life cycle index (linx): A new indexing procedure for process and product design and decision-making. J. Clean. Prod. 2004, 12, 59-76. [CrossRef]

64. Fu, C.; Xu, D.-L. Determining attribute weights to improve solution reliability and its application to selecting leading industries. Ann. Oper. Res. 2016, 245, 401-426. [CrossRef]

65. Fu, C.; Xu, D.-L.; Xue, M. Determining attribute weights for multiple attribute decision analysis with discriminating power in belief distributions. Knowl.-Based Syst. 2018, 143, 127-141. [CrossRef]

66. Xiao, F. A hybrid fuzzy soft sets decision-making method in medical diagnosis. IEEE Access 2018, 6, 25300-25312. [CrossRef]

67. Cao, Z.; Lin, C.T. Inherent fuzzy entropy for the improvement of EEG complexity evaluation. IEEE Trans. Fuzzy Syst. 2018, 26, 1032-1035. [CrossRef]

(C) 2019 by the authors. Licensee MDPI, Basel, Switzerland. This article is an open access article distributed under the terms and conditions of the Creative Commons Attribution (CC BY) license (http://creativecommons.org/licenses/by/4.0/). 